\title{
Safety and tolerability considerations in the use of sildenafil for children with pulmonary arterial hypertension
}

\author{
Andrew L Dodgen' \\ Kevin D Hill',2 \\ 'Department of Pediatrics, Duke \\ University Medical Center, ${ }^{2}$ Duke \\ Clinical Research Institute, Durham, \\ NC, USA
}

This article was published in the following Dove Press journal:

Drug, Healthcare and Patient Safety

15 December 2015

Number of times this article has been viewed

\begin{abstract}
Sildenafil is a phosphodiesterase type-5 inhibitor approved for treatment of pulmonary arterial hypertension (PAH) in adults. Data from pediatric trials demonstrate a similar acute safety profile to the adult population but have raised concerns regarding the safety of long-term use in children. Interpretation of these trials remains controversial with major regulatory agencies differing in their recommendations - the US Food and Drug Administration recommends against the use of sildenafil for treatment of PAH in children, while the European Medicines Agency supports its use at "low doses". Here, we review the available pediatric data regarding dosing, acute, and long-term safety and efficacy of sildenafil for the treatment of PAH in children.
\end{abstract}

Keywords: phosphodiesterase inhibitor, pulmonary vasodilator, STARTS trials

\section{Introduction}

Pulmonary arterial hypertension (PAH) is a disease-causing pathologic changes primarily at the arteriolar level of the pulmonary vascular bed. These changes include development of a vasomotor imbalance favoring pulmonary vasoconstriction, inflammation, and remodeling of the vessel walls. This results in luminal narrowing or obliteration, and possible thrombosis in situ. ${ }^{1-4}$ Ultimately, these factors lead to increased pulmonary vascular resistance with secondarily increased pulmonary arterial pressures. When pulmonary vascular resistance and pressures are significantly elevated, right ventricular dysfunction ensues with associated low cardiac output. It is these hemodynamic consequences that cause the progressive and debilitating clinical symptoms of PAH.

Historically, survival for patients diagnosed with PAH has been poor. However, outcomes have improved over the past 3 decades following the development of targeted PAH agents. Only two of these agents have been studied in children in rigorous randomized controlled trials: inhaled nitric oxide (NO), which can only be administered in an intensive care setting, and sildenafil. Sildenafil was studied in the Sildenafil in Treatment-Naïve Children, Aged 1 to 17 Years, With Pulmonary Arterial Hypertension (STARTS) trial, a multicenter, multinational, randomized, and placebo-controlled trial in children with $\mathrm{PAH}^{5,6}$ The results of this trial and the STARTS-II extension trial have been controversial with interpretations ranging from "safe and efficacious" to "dangerous and ineffective". ${ }^{7-9}$ Here we will review the clinical presentation, diagnosis, and natural history of PAH and then summarize available data on safety and efficacy of sildenafil in adults and children.

\section{Clinical presentation and diagnosis}

Patients affected with PAH often present with nonspecific symptoms, which can make clinical diagnosis difficult, sometimes leading to delays in diagnosis. ${ }^{10,11}$ 
Another diagnostic challenge is the significant heterogeneity in age at presentation and in symptoms in children of different ages. In the prospective Tracking Outcomes and Practice in Pediatric Pulmonary Hypertension registry the median age at presentation for pediatric $\mathrm{PAH}$ was 8 years, however, $17 \%$ of patients presented before 2 years of age and $28 \%$ presented during adolescence. ${ }^{10}$ Among infants and younger children, the clinical presentation of PAH may consist of failure to thrive, tachypnea, or symptoms of compromised cardiac output such as feeding intolerance. Older children and adolescents typically present with excessive fatigue, exercise intolerance, syncope or near syncope, and occasionally chest pain. ${ }^{10-13}$

Given the nonspecific nature of clinical symptoms, accurate hemodynamic assessment is recommended for diagnosis. There are several noninvasive methods to estimate pulmonary artery pressures, including echocardiography and cardiac magnetic resonance imaging. However, cardiac catheterization remains the gold standard diagnostic test. Diagnostic criteria for PAH include a mean pulmonary artery pressure $\geq 25 \mathrm{mmHg}$ in the setting of a mean pulmonary capillary wedge pressure of $\leq 15 \mathrm{mmHg} .^{1,13-15}$ In younger children and in those with associated intracardiac shunts, pulmonary arterial pressures alone may inaccurately represent severity of disease. Therefore, pulmonary arterial pressures are often compared to systemic pressures and a pulmonary vascular resistance index above 3 Wood Units $\times \mathrm{m}^{2}$ is typically also considered necessary for diagnosis. ${ }^{14}$

\section{Classification and epidemiology}

PAH represents a distinct category of pulmonary hypertension that must be distinguished from other causes of elevated pulmonary arterial pressures including left heart diseases (eg, cardiomyopathy or mitral stenosis), lung diseases (eg, chronic obstructive pulmonary disease or obstructive sleep apnea), and chronic thromboembolic pulmonary hypertension. The most recent guidelines for classification of pulmonary hypertension, updated by the Task Force for the Diagnosis and Treatment of Pulmonary Hypertension at the Fifth World Symposium on Pulmonary Hypertension in Nice, France in 2013, identify PAH as "Group I". Within this group are several subcategories of $\mathrm{PAH}$, including idiopathic $\mathrm{PAH}$, heritable $\mathrm{PAH}$, drug- and toxin-induced $\mathrm{PAH}$, conditions with histopathological changes of idiopathic PAH and a similar clinical presentation, such as connective tissue disease and congenital heart disease. ${ }^{15}$

Accurate classification of PAH (Group I pulmonary hypertension) and differentiation from other forms of pulmonary hypertension has important epidemiologic and therapeutic implications. For example, pulmonary vasodilator therapy is the mainstay of treatment for adults with PAH (Group I), but there is evidence to suggest that potent pulmonary vasodilator therapy can be harmful in Group II pulmonary hypertension (ie, associated with left heart disease). ${ }^{16}$ Moreover, the benefits have not been well studied in children with pulmonary hypertension associated with lung diseases (Group III). Overall PAH is a rare disease in children. In a retrospective cohort from the Netherlands, the reported annual incidence was 3.0 cases per million children including 2.2 cases per million children for $\mathrm{PAH}$ related to congenital heart disease and 0.7 cases per million for idiopathic PAH. ${ }^{17} \mathrm{~A}$ similar retrospective registrybased study out of the UK reported an annual incidence of idiopathic PAH of 0.48 cases per million children. ${ }^{18}$ The pathophysiology of pediatric $\mathrm{PAH}$ also differs from $\mathrm{PAH}$ in adults where entities like collagen vascular disease and portal hypertension account for $\sim 25 \%$ of cases. In contrast, these subgroups are uncommon in children, accounting for less than $2 \%$ of all cases in the multinational Tracking Outcomes and Practice in Pediatric Pulmonary Hypertension registry where $57 \%$ of $\mathrm{PAH}$ cases were identified as having idiopathic or familial PAH, while $38 \%$ had PAH associated with congenital heart disease. ${ }^{10}$

\section{Outcomes}

Untreated, PAH leads to progressive clinical deterioration and ultimately death from right heart failure. Historical data indicate that PAH outcomes among children have been quite poor, with survival rates beyond 1 and 7 years in 1965 having been reported as $37 \%$ and $0 \%$, respectively. ${ }^{19}$ Over the course of the next several decades survival rates improved, with reported 1-, 3-, and 5-year survival rates from the 1950s to 1990 s of $66 \%, 52 \%$, and $35 \%$, respectively. ${ }^{11,12,20}$ However, prior to the development of targeted therapies, and as recently as 1995 , the median survival time following diagnosis of PAH was still very poor at just over 4 years. ${ }^{20}$ Concurrent with the advent of targeted therapies for PAH, reported survival rates have improved; $72 \%-74 \%$ at 5 years in contemporary analyses from the UK and the USA. ${ }^{18,21}$

\section{Treatment of PAH}

Historically, there have been very few rigorous clinical trials of PAH therapies in children. Even relatively smaller and easier to conduct pharmacokinetic-pharmacodynamic studies have been limited in this population and instead dosing, safety, and efficacy of drugs have been extrapolated 
from adult trials. This practice is no longer considered acceptable as children demonstrate important differences in disease pathophysiology and pharmacokinetic to adults. ${ }^{22}$ Accordingly, new agents to treat pediatric pulmonary hypertension have been identified as a high priority for study by the US Food and Drug Administration (FDA) and are listed on the Best Pharmaceuticals for Children's Act (BPCA) Priority List of Needs in Pediatric Therapeutics. ${ }^{23}$ BPCA created incentives for studying on-patent drugs in children, most notably, a potential 6-month patent extension. The financial gains associated with patent extension are potentially very significant and so industry sponsors have been willing to invest substantially in pediatric clinical trials. ${ }^{24}$ Sildenafil was the first outpatient therapeutic agent studied for treatment of pediatric PAH under the auspices of BPCA (the STARTS-I and STARTS-II trials). ${ }^{5,6}$ These studies have contributed a wealth of data but, like many clinical trials, have also created controversy. In the following sections, we will review the mechanism of action of sildenafil and other phosphodiesterase type-5 (PDE-5) inhibitors and summarize the available data from the STARTS trials as well as other studies evaluating pharmacokinetic-pharmacodynamic, safety and efficacy of sildenafil.

\section{Sildenafil mechanism of action}

Sildenafil is a selective PDE-5 inhibitor and potentiates the downstream effects of NO. NO induces smooth muscle relaxation and vasodilation through its effects on the cyclic guanosine monophosphate (c-GMP) pathway. ${ }^{2,25}$ Specifically, NO binds to and activates guanylyl cyclase in vascular smooth muscle cells. This enzyme then catalyzes the conversion of guanosine triphosphate to c-GMP, which, in turn, acts as a second messenger mediating several cellular processes including smooth muscle relaxation. . $^{2,25}$

PDE-5 limits the vasodilating effects of NO by degrading c-GMP and is selectively expressed in the pulmonary vasculature, as well as the corpora cavernosa. Therefore, sildenafil and other PDE-5 inhibitors are relatively selective pulmonary vasodilators. ${ }^{2,25}$ Additionally, sildenafil and other PDE-5 inhibitors have the added benefit of exerting an antiproliferative effect within the pulmonary vascular bed, thereby addressing a second pathophysiologic mechanism of $\mathrm{PAH}{ }^{26,27}$

\section{Sildenafil formulations, pharmacokinetics, and drug interactions}

Sildenafil (marketed for PAH under the brand name Revatio ${ }^{\circledR}$, Pfizer, Inc, New York, NY, USA) is available as a $20 \mathrm{mg}$ film-coated tablet, a $10 \mathrm{mg} / 12.5 \mathrm{~mL}$ vial for injection, and as a powder for reconstitution into a liquid suspension (with instructions on the package insert). The recommended dosing interval is three times a day (tid) with maximum plasma concentrations observed within 30-120 minutes (median 60 minutes) of oral dosing in the fasted state. The mean absolute bioavailability for the tablet is $41 \%$ (range $25 \%-63 \%$ ) and the recommended intravenous dose is half that of the oral dose. $^{28}$

Sildenafil is almost exclusively metabolized in the liver and there are important dosing considerations when prescribing sildenafil to children, particularly neonates and patients with hepatic dysfunction. In the first few days of life, clearance of sildenafil is approximately one-third of that seen in adults with a markedly prolonged half-life of 48-56 hours (vs 2.2-3.9 hours in healthy adults). This has been attributed to immaturity of the cytochrome P450 (CYP) metabolizing system, which undergoes rapid maturation in the first week of life with clearance increasing accordingly. ${ }^{29-31}$ Additionally, hepatic metabolism of sildenafil can vary depending on the hemodynamic conditions under which it is administered. Elevated hepatic pressures, hepatic congestion, and hepatic cirrhosis have all been shown to delay sildenafil clearance and increase drug exposure. ${ }^{32-35}$

Sildenafil clearance is also affected by agents that induce or inhibit major CYP isoforms. ${ }^{36}$ Perhaps most commonly this is an issue with coadministration of bosentan, a known CYP3A4 inducer frequently used in combination with sildenafil for treatment of PAH. Bosentan accelerates sildenafil clearance and reduces sildenafil concentrations by $\sim 60 \%$ when used at standard doses. ${ }^{37}$ Caution is also warranted when using medications with similar side effect profiles or mechanisms of action to sildenafil. ${ }^{36}$ Most notably, medications with hypotensive effects have the potential to induce severe hypotension when administered in combination with sildenafil. For example, given that both exert their therapeutic effects by increasing the activity of c-GMP, the combination of sildenafil and organic nitrates is generally contraindicated. ${ }^{38}$

\section{Sildenafil efficacy and tolerability in adults with $\mathrm{PAH}$}

Perhaps the most notable sildenafil trials conducted in the adult population are the double-blinded, placebo-controlled sildenafil use in PAH study, or Sildenafil Use in Pulmonary Arterial Hypertension (SUPER)-1 study, and its extension study, SUPER-2. ${ }^{25,39}$ In the SUPER-1 trial, sildenafil use in adult patients with idiopathic PAH or PAH associated with 
connective tissue or congenital heart disease was associated with enhanced exercise capacity and improved hemodynamics after 12 weeks of therapy. Assessment of exercise capacity in this study was based on the 6-minute walk test, with a dose-related increase in the average distance walked by $13 \%$ (average improvement of $38 \mathrm{~m} ; 99 \%$ confidence interval [CI]: 12-64 m), 13.3\% (average improvement of $45 \mathrm{~m} ; 99 \%$ CI: 21-70 m), and 14.7\% (average improvement of $42 \mathrm{~m}$; 99\% CI: 9-75 m), for sildenafil tid doses of 20, 40, or 80 $\mathrm{mg}$, respectively. ${ }^{25}$

The SUPER-2 extension trial evaluated the long-term safety and tolerability of sildenafil for the treatment of PAH, enrolling 93\% of SUPER-1 participants. Each patient enrolled in SUPER-2 was titrated to the maximum tolerable tid dose of sildenafil (up to $80 \mathrm{mg}$ per dose) with 6-minute walk assessed at each scheduled visit. At the end of the 3-year study period, $87 \%$ of enrollees who remained on treatment were receiving $80 \mathrm{mg}$ of sildenafil tid, while $8 \%$ were receiving $40 \mathrm{mg}$ tid, and 5\% were receiving $20 \mathrm{mg}$ tid. Overall 39 (14\%) subjects discontinued treatment due to known or perceived sildenafil-related adverse effects and 59 (22\%) died. Of the 277 patients who completed SUPER-1, 31\% improved and 29\% maintained their baseline New York Heart Association (NYHA) functional class at 3-year follow-up. ${ }^{39}$

In addition to the SUPER-1 and SUPER-2 trials, several other randomized controlled trials have evaluated efficacy of sildenafil for treatment of PAH in adults. In the 16-week Pulmonary Arterial Hypertension Combination Study of Epoprostenol and Sildenafil (PACES) study, 267 patients with PAH receiving long-term intravenous epoprostenol were randomized to placebo or sildenafil, dosed at $20 \mathrm{mg}$ tid and titrated up to a maximum of $80 \mathrm{mg}$ tid. ${ }^{40}$ Compared to epoprostenol alone, subjects also receiving sildenafil demonstrated increased 6-minute walk distance (+28.8 m, 95\% CI: $13.9-43.8 \mathrm{~m})$, decreased mean pulmonary artery pressure $(-3.8 \mathrm{mmHg}, 95 \% \mathrm{CI}:-5.6$ to $-2.1 \mathrm{mmHg})$, and increased cardiac output $(+0.9 \mathrm{~L} / \mathrm{min}, 95 \% \mathrm{CI}: 0.5-1.2 \mathrm{~L} / \mathrm{min})$. In a smaller crossover trial, Sastry et $\mathrm{al}^{41}$ randomized 22 patients to placebo versus sildenafil (dose ranging from 25 to $100 \mathrm{mg}$ tid) for a total of 6 weeks. After completion of the initial 6 weeks of therapy, each participant then crossed over to the alternative therapy to complete an additional 6 weeks of therapy. Sildenafil therapy was associated with improvements in both exercise time (improved by $44 \%, P<0.0001$ ) and cardiac index (improved from $2.80 \pm 0.9$ to $3.45 \pm 1.1$ $\left.\mathrm{L} / \mathrm{m}^{2}, P<0.0001\right){ }^{41}$

Numerous trials of other pulmonary vasodilator therapies have been completed in adults with PAH. At least one meta-analysis of the available randomized controlled trials has been performed. The main outcome measure of this analysis was all-cause mortality and a total of 23 randomized controlled trials were included with an average duration of 14.3 weeks. The results of this analysis demonstrated a $43 \%$ reduction in all-cause mortality, as well as a $61 \%$ reduction in hospitalizations in patients undergoing targeted pharmacologic treatment of PAH compared to patients receiving placebo. ${ }^{42}$

\section{The STARTS trials}

Positive results from adult studies of pulmonary vasodilator therapies, coupled with the availability of appropriate pediatric sildenafil preparations, prompted numerous smaller, mostly single-center reports describing positive outcomes with off-label use of sildenafil. However, sildenafil had not been well studied for pediatric PAH (with the possible exception of neonates with persistent pulmonary hypertension of the newborn (PPHN) - see "Sildenafil for PPHN" section) until the STARTS trials. STARTS-I was a randomized, double-blind, placebo-controlled, dose-ranging study on the use of oral sildenafil monotherapy for PAH in treatment-naïve children. ${ }^{6} \mathrm{~A}$ total of 235 children aged 1-17 years were enrolled in the study and randomized to placebo, low-, medium-, or high-dose sildenafil administered tid for a total of 16 weeks. Administered dosages varied by weight; for patients $<45 \mathrm{~kg}$, doses were calculated to achieve target plasma concentrations based on scaled adult pharmacokinetic data (targeting peak plasma concentrations of 47, 140, and $373 \mathrm{ng} / \mathrm{mL}$ for low-, medium-, and high-dose groups, respectively). For subjects weighing $>45 \mathrm{~kg}$, the dosing groups mirrored those used in the SUPER-1 trials in adults (10, 40, and 80 mg for low-, medium-, and high-dose groups, respectively). The primary outcome measure was percent change in peak oxygen consumption in all patients receiving sildenafil combined versus placebo, with exercise testing being performed in all patients able to exercise reliably. Although the study ultimately enrolled 235 subjects, it was expected that many of the younger patients would not be able to complete exercise testing (yet were enrolled for the purposes of safety follow-up). The investigators accounted for this limitation in their sample size estimates; an enrollment of 104 subjects who could complete exercise testing was targeted (ultimately 106 were enrolled) to provide $90 \%$ power to demonstrate a $15 \%$ improvement in peak oxygen consumption for the combined sildenafil treatment groups relative to placebo. The decision was made to combine the sildenafil treatment groups for the purposes of this analysis 
as adult data indicated a lack of dose-related improvement in 6-minute walk test results. By combining the sildenafil dose groups, the authors were able to reduce the sample size and treatment effect size on which to base the study.

Results demonstrated a percent change of $7.7 \% \pm 4.0 \%$ in peak oxygen consumption in the combined sildenafil group with a nonsignificant $P$-value of 0.056 (95\% CI, $-0.2 \%$ to $15.6 \%$ ). Patients with idiopathic PAH demonstrated greater improvements than patients with associated PAH but these improvements did not reach statistical significance for either of these subgroups (note: it is likely that these subgroup analyses were underpowered). Secondary endpoints, included change from baseline to week 16 in mean hemodynamics, functional class, physical, and psychosocial scales of the Child Health Questionnaire-Parent Form 28 (for patients aged $\geq 5$ years), and percent change from baseline in exercise duration. These secondary endpoints were assessed in all patients enrolled in the trial and some of these improved in select subgroups when compared to placebo. However, patients receiving low-dose sildenafil demonstrated no significant improvement in any of the secondary outcome measures (Table 1).,

In the STARTS-II extension trial, all STARTS-I placebo enrollees were re-randomized to low-, medium-, or highdose sildenafil, with those having received sildenafil during STARTS-I continuing their previously assigned dose. Dose titration was permitted throughout the STARTS-II trial at the discretion of the investigator. After 3 years of treatment, a total of 37 patients had died, 26 of which remained on sildenafil monotherapy at the time of death. No deaths occurred in the first 16 weeks of therapy and only one occurred in the first year of the trial; therefore, all deaths occurred in patients who had been assigned to a sildenafil treatment group. Based on their original treatment assignment, deaths occurred in 5/55 (9\%), 10/74 (14\%), and 22/100 (22\%) for low-, medium-, and high-dose groups, respectively, with estimated 3-year survival of $94 \%, 93 \%$, and $88 \%$ for the sequential dosing groups (Figure 1). ${ }^{5,6}$ Hazard ratios for mortality were 3.95 (95\% CI, 1.46-10.65) for high-dose sildenafil use compared to low-dose sildenafil and 1.92 for medium-dose sildenafil compared to low-dose sildenafil (95\% CI, 0.65-5.65). These results prompted the US FDA to issue a recommendation against the use of sildenafil for $\mathrm{PAH}$ in pediatric patients, citing increased mortality risk with high-dose use and no evidence to support efficacy with low-dose use. ${ }^{8}$ However, the European Medicines Agency (EMA) reviewed identical data from the STARTS trials but determined that the available data supported the use of sildenafil in pediatric patients. ${ }^{7}$ The EMA approved sildenafil at oral doses of $10 \mathrm{mg}$ tid for children $<20 \mathrm{~kg}$ and $20 \mathrm{mg}$ tid for children $\geq 20 \mathrm{~kg}$. For children $<45 \mathrm{~kg}$, these recommendations correspond to the mediumdose groups in the STARTS trial, while for children $\geq 45 \mathrm{~kg}$, they correspond to the low-dose groups. The US FDA later released a clarification of their original recommendations, stating that there may be situations in which the risks of sildenafil use may be acceptable in individual children.

The disparate opinions of the FDA and EMA stem from several inconsistencies in the data, as well as from inherent difficulties in conducting clinical trials in children with a rare disease:

- First, the study cohort consisted of a heterogeneous collection of patients including a broad range of ages (1-17 years), diagnoses (33\% had idiopathic PAH/heritable PAH and $67 \%$ had PAH associated with congenital heart disease) and disease severity (World Health Organization functional class I, II, III, and IV). Trials that enroll heterogeneous patient cohorts can be difficult to analyze and in the subanalyses of the STARTS trials, there were discrepancies in mortality rates across weight ranges, by functional class

Table I Secondary and tertiary outcomes evaluated in the STARTS-I trial

\begin{tabular}{|c|c|c|c|c|}
\hline & Low dose & Medium dose & High dose & Combined \\
\hline Peak $\mathrm{VO}_{2}$ (\% change from baseline) & $3.8(-6.1,13.7)$ & II.3 (-I.7, 20.9) & $8.0(-1.6,17.6)$ & $7.7(-0.2,15.6)$ \\
\hline Mean PA pressure ( $\mathrm{mmHg}$ change from baseline) & $1.6(-4.5,7.6)$ & $-3.5(-8.9,1.9)$ & $-7.3(-12.4,-2.1)^{*}$ & $-3.1(-7.5,1.3)$ \\
\hline PVRI (ratio I6 weeks to baseline) & $0.98(0.80,1.20)$ & $0.82(0.68,0.98)^{*}$ & $0.73(0.61,0.86)^{*}$ & $0.84(0.72,0.97)^{*}$ \\
\hline RA pressure (mmHg change from baseline) & $-0.2(-1.9,1.6)$ & $-0.2(-1.7,1.4)$ & I.I $(-2.6,0.3)$ & $-0.5(-1.8,0.8)$ \\
\hline Cardiac index ratio (I6 weeks to baseline) & I.I $(0.96,1.26)$ & $1.04(0.93,1.18)$ & $1.15(1.03,1.29)^{*}$ & $\mathrm{I} .10(0.99, \mathrm{I} .2 \mathrm{I})$ \\
\hline Exercise duration (\% change from baseline) & $10.3(-5.2,25.9)$ & II.4 (-3.8, 26.6) & $6.0(-9.2,21.1)$ & $9.2(-3.1,21.5)$ \\
\hline Functional class (odds of improvement vs placebo) & $0.6(0.2-2.0)$ & $2.3(0.8-6.7)$ & $4.5(1.6-13.1)^{*}$ & No change \\
\hline Patient/parent reported global improvement (\%) & $35.7^{*}$ & $34.6 *$ & $45.5^{*}$ & Improved* \\
\hline Physician reported global improvement (\%) ${ }^{\mathrm{a}}$ & $26.2^{*}$ & $27.2 *$ & $28.6 *$ & Improved* \\
\hline
\end{tabular}

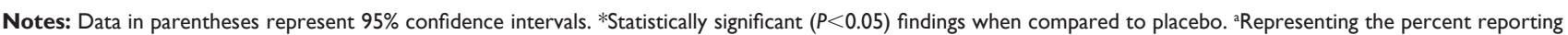
moderate or marked improvement versus $21.6 \%$ of patient/parents and $10 \%$ of physicians reporting improvement for the placebo group. Absolute values not available for the combined treatment groups.

Abbreviations: PA, pulmonary arterial; RA, right atrial; vs, versus; PVRI, pulmonary vascular resistance index; STARTS, Sildenafil in Treatment-Naïve Children, Aged I to 17 Years, With Pulmonary Arterial Hypertension; $\mathrm{VO}_{2}$, oxygen consumption. 


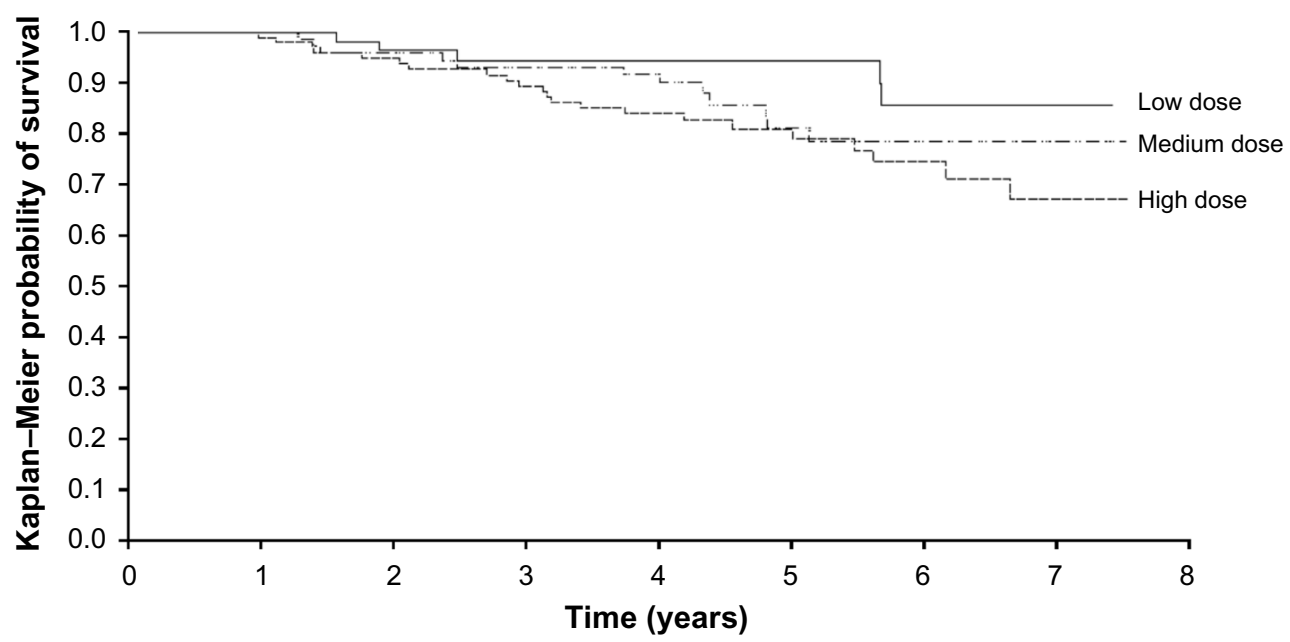

Figure I Long-term survival for children treated with sildenafil in the STARTS trials. Note: Data from the US Food and Drug Administration.'

Abbreviation: STARTS, Sildenafil in Treatment-Naïve Children, Aged I to 17 Years, With Pulmonary Arterial Hypertension.

and by diagnostic cohorts. Most notably, patients with idiopathic PAH/heritable PAH accounted for $77 \%$ of all deaths but only $33 \%$ of the study population., 5

- Second, despite randomization the placebo group appeared to have better baseline hemodynamics with lower pulmonary vascular resistance than the combined treatment cohort $\left(15 \pm 10\right.$ Wood Units $\times \mathrm{m}^{2}$ vs $20 \pm 15$ Wood Units $\times \mathrm{m}^{2}$ ). Moreover, subjects who received placebo in the initial study and then went on to receive sildenafil in the long-term extension had better survival than those who received sildenafil from the start of the trial, suggesting that a 16-week delay in the start of sildenafil treatment is beneficial for survival. There is no physiologically plausible explanation as to why delay in treatment by 16 weeks would confer a long-term survival advantage. ${ }^{5}$

- Third, the highest mortality rates were observed in a small subset of developing countries, with rates of $44 \%, 36 \%$, and $21 \%$ occurring in India, Mexico, and Poland, respectively, while a rate of only $5 \%$ was observed in the USA. ${ }^{5,9}$

- Fourth, most deaths were deemed by the investigators to be related to disease progression and none of the observed deaths were deemed attributable to study treatment., ${ }^{5,79}$

- Fifth, there were inconsistencies in the clinical pharmacology data with an exposure-dependent increase in mortality noted in the low- and medium-dose groups, but a trend in the opposite direction in the high-dose group. ${ }^{5,7,9}$

- Finally, critics of the US FDA opinion note that the entire treatment cohort had improved mortality rates when compared to the "untreated" natural history of PAH. Indeed, the STARTS-II investigators offered an alternative hypothesis to the interpretation that high-dose sildenafil is associated with increased mortality, postulating that low-dose sildenafil is associated with an improved survival rate and that this treatment benefit diminishes at higher doses. ${ }^{5,7,9}$

In summary, it is reasonable to conclude that the higher doses used in the STARTS trials are associated with worse outcomes than the lower dose groups. However, controversy persists over whether the potential clinical benefit of low-dose sildenafil use in pediatric PAH justifies the potential risks of use. Continued research is needed and will likely be required prior to any change in the US FDA recommendation against its use in children.

\section{Sildenafil for PPHN}

Sildenafil has also been studied rigorously in the treatment of PPHN. ${ }^{43}$ In a dose-ranging trial, a total of 36 neonates were assigned to one of eight sequential sildenafil dosing groups and underwent continuous treatment with intravenous sildenafil for a minimum of 48 hours. Baseline oxygenation index was calculated for each patient and then repeated at assigned intervals during the course of treatment. For the study group as a whole, the oxygenation index was significantly improved from baseline measurements over the first 24 hours of treatment $(27.7 \pm 4.2$ to $11.3 \pm 2.3 ; P<0.0001)$. Additionally, a dose-related effect was seen, with groups 1-3 (mean initial sildenafil plasma concentration of $3.7 \pm 4.6 \mathrm{ng} / \mathrm{mL}$ ) experiencing no change from baseline oxygenation index over the initial 4 hours of treatment while groups $4-8$ (mean initial sildenafil plasma concentration of $58.4 \pm 44.8 \mathrm{ng} / \mathrm{mL})$ experienced a significant improvement over the same time period ( $29 \pm 6$ to $19 \pm 6 ; P=0.0002)$. Six treatment-related adverse events were reported during the study and ranged from mild to moderate 
in terms of severity, including hypotension, blood pressure lability, and development of a Patent Ductus Arteriosus (PDA) that resolved without intervention. ${ }^{43}$

\section{Sildenafil after Fontan surgery}

Other relatively more rigorous studies regarding the use of sildenafil in children have dealt with specific subsets of the pediatric population, including patients with congenital heart disease who have undergone complete Fontan palliation. In a double-blind, placebo-controlled, crossover trial in children and young adults (mean age 14.9 \pm 5.1 years) following Fontan surgery, subjects were randomized to receive placebo or sildenafil (20 mg tid) for 6 weeks. ${ }^{44}$ This was followed by a 6-week washout period, after which subjects crossed over to receive the alternate therapy for an additional 6 weeks. Exercise stress testing was performed at the beginning and end of each phase and the primary endpoint of oxygen consumption at peak exercise was assessed. Following sildenafil no change in oxygen consumption during peak exercise was observed. However, subjects had improvements in measures of ventilatory efficiency, including a significantly decreased respiratory rate and decreased minute ventilation at peak exercise. ${ }^{44}$ The lack of a significant improvement in the primary outcome measure differs from results in the adult Fontan population where peak oxygen consumption improved among Fontan patients receiving sildenafil compared to placebo 1 hour following dose administration, although the study design was not as rigorous as the aforementioned crossover trial in adolescents. $^{45}$

\section{Safety considerations}

Adverse effects associated with sildenafil use in children have been described in both the STARTS-I and STARTS-II trials, and at least one dedicated study of pediatric tolerability. $5,6,46$ Reported adverse effects seen in children are mostly of mildto-moderate severity, similar to those observed in adults (Table 2) and to the premarket sildenafil trials. ${ }^{25,28,39}$ Treatment-related serious adverse effects have been uncommon in the pediatric trials, occurring in a total of two STARTS-I enrollees and five STARTS-II enrollees, and including hypersensitivity and stridor $(n=2)$, ventricular arrhythmia $(n=2)$, enterocolitis $(n=1)$, hypoxia $(\mathrm{n}=1)$, and convulsions $(\mathrm{n}=1)$.

\section{Conclusion}

$\mathrm{PAH}$ is a rare but serious disease in children with a historically poor prognosis. Tremendous strides have been made in the treatment of PAH over the past several decades including the development of numerous targeted PAH agents. Although these agents have all been rigorously studied in adults with
Table 2 Frequency of adverse events associated with sildenafil use in adults as reported on the US Food and Drug Administration Drug Label

\begin{tabular}{lll}
\hline Adverse Event & $\begin{array}{l}\text { Percentage of patients reporting } \\
\text { event }\end{array}$ \\
\hline & Sildenafil (n=70) & Placebo (n=69) \\
\hline Epistaxis & $9 \%$ & $1 \%$ \\
Headache & $46 \%$ & $39 \%$ \\
Flushing & $10 \%$ & $4 \%$ \\
Insomnia & $7 \%$ & $1 \%$ \\
Erythema & $6 \%$ & $1 \%$ \\
Dyspnea exacerbated & $7 \%$ & $3 \%$ \\
Dyspepsia & $13 \%$ & $7 \%$ \\
Rhinitis & $4 \%$ & $0 \%$ \\
Diarrhea & $9 \%$ & $6 \%$ \\
Myalgia & $7 \%$ & $3 \%$ \\
Pyrexia & $6 \%$ & $3 \%$ \\
Gastritis & $3 \%$ & $0 \%$ \\
Sinusitis & $3 \%$ & $0 \%$ \\
Paresthesia & $3 \%$ & $0 \%$ \\
\hline
\end{tabular}

Notes: Reproduced from the US Food and Drug Administration. ${ }^{47}$ Available from: http://www.accessdata.fda.gov/drugsatfda_docs/label/2014/02 I845s0 I I,022473s004,

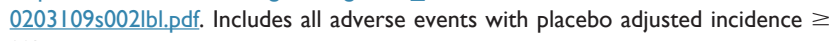
$3 \%$; adverse events reported in adult subjects with pulmonary arterial hypertension taking doses of $20 \mathrm{mg}$ three times daily.

favorable safety and efficacy profiles, very few studies have been conducted specifically in children with PAH. Sildenafil, a PDE-5 inhibitor approved for treatment of PAH in adults, was the first chronic PAH therapy to be studied in a rigorous fashion in children in the STARTS-I and STARTS-II trials. In these trials, sildenafil demonstrated a similar acute adverse effect profile to that seen in adult populations, however, lowdose sildenafil failed to demonstrate significant improvement in the primary trial endpoint when compared to placebo and high-dose sildenafil was associated with increased mortality at long-term follow-up. Interpretation of these trials has proven very difficult due to several inconsistencies in the data. Although most agree that high doses of sildenafil should be avoided in children, even major regulatory agencies differ in their recommendations regarding lower doses of sildenafil; the EMA supports use at lower doses, while the US FDA recommends against use of sildenafil at any dose in children. Further studies are needed to clarify the long-term safety and efficacy of sildenafil as well as other targeted PAH agents for use in children. These studies can benefit from lessons learned in the STARTS trials, including the critical importance of powering studies for stratified analysis based on age and underlying PAH diagnosis.

\section{Acknowledgments}

$\mathrm{KDH}$ is supported in part by a grant KL2TR001115-02 from The National Center for Advancing Translational Sciences of the National Institutes of Health, and a grant from the Mend 
A Heart Foundation. The content is solely the responsibility of the authors and does not necessarily represent the official views of the National Institutes of Health or other funding groups.

\section{Disclosure}

The authors report no conflicts of interest in this work.

\section{References}

1. Galie N, Hoeper MM, Humbert M, et al. Guidelines for the diagnosis and treatment of pulmonary hypertension: the Task Force for the Diagnosis and Treatment of Pulmonary Hypertension of the European Society of Cardiology (ESC) and the European Respiratory Society (ERS), endorsed by the International Society of Heart and Lung Transplantation (ISHLT). Eur Heart J. 2009;30(20):2493-2537.

2. Humbert M, Sitbon O, Simonneau G. Treatment of pulmonary arterial hypertension. N Engl J Med. 2004;351(14):1425-1436.

3. Latus H, Delhaas T, Schranz D, Apitz C. Treatment of pulmonary arterial hypertension in children. Nat Rev Cardiol. 2015;12(4):244-254.

4. Vorhies EE, Ivy DD. Drug treatment of pulmonary hypertension in children. Paediatr Drugs. 2014;16(1):43-65.

5. Barst RJ, Beghetti M, Pulido T, et al. STARTS-2: long-term survival with oral sildenafil monotherapy in treatment-naive pediatric pulmonary arterial hypertension. Circulation. 2014;129(19):1914-1923.

6. Barst RJ, Ivy DD, Gaitan G, et al. A randomized, double-blind, placebocontrolled, dose-ranging study of oral sildenafil citrate in treatmentnaive children with pulmonary arterial hypertension. Circulation. 2012;125(2):324-334.

7. European Medicines Agency Science Medicines Health Assessment report for Revatio. Available from: http://www.ema.europa.eu/docs/ en_GB/document_library/EPAR_-_Assessment_Report_-_Variation/ human/000638/WC500107804.pdf. Accessed April 14, 2015.

8. US Food and Drug Administration. Drug safety communication. Available from: http://www.fda.gov/Drugs/DrugSafety/ucm390876. htm. Accessed April 14, 2015.

9. US Food and Drug Administration. Medical, statistical, and clinical pharmacology reviews of pediatric studies conducted under Section 505A and 505B of the Federal Food, drug, and Cosmetic Act, as amended by the FDA Amendments Act of 2012 (FDASIA). Available from: http://www.fda.gov/Drugs/DevelopmentApprovalProcess/Devel opmentResources/ucm316937.htm. Accessed April 14, 2015.

10. Berger RM, Beghetti M, Humpl T, et al. Clinical features of paediatric pulmonary hypertension: a registry study. Lancet. 2012;379(9815): 537-546.

11. Ivy DD, Abman SH, Barst RJ, et al. Pediatric pulmonary hypertension. J Am Coll Cardiol. 2013;62(25 Suppl):D117-D126.

12. Barst RJ, Ertel SI, Beghetti M, Ivy DD. Pulmonary arterial hypertension: a comparison between children and adults. Eur Respir J. 2011;37(3): 665-677.

13. van Loon RL, Roofthooft MT, van Osch-Gevers M, et al. Clinical characterization of pediatric pulmonary hypertension: complex presentation and diagnosis. J Pediatr. 2009;155(2):176-182. e171.

14. Hill KD, Lim DS, Everett AD, Ivy DD, Moore JD. Assessment of pulmonary hypertension in the pediatric catheterization laboratory: current insights from the Magic registry. Catheter Cardiovasc Interv. 2010;76(6):865-873.

15. Simonneau G, Gatzoulis MA, Adatia I, et al. Updated clinical classification of pulmonary hypertension. J Am Coll Cardiol. 2013; 62(25 Suppl):D34-D41.

16. Califf RM, Adams KF, McKenna WJ, et al. A randomized controlled trial of epoprostenol therapy for severe congestive heart failure: the Flolan International Randomized Survival Trial (FIRST). Am Heart J. 1997;134(1):44-54.
17. van Loon RL, Roofthooft MT, Hillege HL, et al. Pediatric pulmonary hypertension in the Netherlands: epidemiology and characterization during the period 1991 to 2005. Circulation. 2011;124(16):1755-1764.

18. Haworth SG, Hislop AA. Treatment and survival in children with pulmonary arterial hypertension: the UK Pulmonary Hypertension Service for Children 2001-2006. Heart. 2009;95(4):312-317.

19. Thilenius OG, Nadas AS, Jockin H. Primary pulmonary vascular obstruction in children. Pediatrics. 1965;36:75-87.

20. Sandoval J, Bauerle O, Gomez A, Palomar A, Martinez Guerra ML, Furuya ME. Primary pulmonary hypertension in children: clinical characterization and survival. J Am Coll Cardiol. 1995;25(2):466-474.

21. Barst RJ, McGoon MD, Elliott CG, Foreman AJ, Miller DP, Ivy DD. Survival in childhood pulmonary arterial hypertension: insights from the registry to evaluate early and long-term pulmonary arterial hypertension disease management. Circulation. 2012;125(1):113-122.

22. Hill KD, Smith PB, Cohen-Wolkowiez M, Benjamin DK, Li JS. Pediatric exclusivity and other contemporary regulatory changes: impact on pediatric drug study, labeling and safety. Clin Investig. 2013;3(13): $227-229$.

23. United States National Institutes of Health. Best Pharmaceuticals for Children Act (BPCA) Priority List of Needs in Pediatric Therapeutics. Available from: http://bpca.nichd.nih.gov/prioritization/status/ documents/priority_list_10-26-2012.pdf. Accessed May 5, 2015.

24. Li JS, Eisenstein EL, Grabowski HG, et al. Economic return of clinical trials performed under the pediatric exclusivity program. JAMA. 2007; 297(5):480-488.

25. Galie N, Ghofrani HA, Torbicki A, et al. Sildenafil citrate therapy for pulmonary arterial hypertension. $N$ Engl J Med. 2005;353(20): $2148-2157$.

26. Tantini B, Manes A, Fiumana E, et al. Antiproliferative effect of sildenafil on human pulmonary artery smooth muscle cells. Basic Res Cardiol. 2005;100(2):131-138.

27. Wharton J, Strange JW, Moller GM, et al. Antiproliferative effects of phosphodiesterase type 5 inhibition in human pulmonary artery cells. Am J Respir Crit Care Med. 2005;172(1):105-113.

28. Revatio ${ }^{\circledR}$ [package insert]. New York, NY: Pfizer Labs; 2014. Available from: http://labeling.pfizer.com/ShowLabeling. aspx?id=645. Accessed May 5, 2015.

29. Koukouritaki SB, Manro JR, Marsh SA, et al. Developmental expression of human hepatic CYP2C9 and CYP2C19.J Pharmacol Exp Ther. 2004;308(3):965-974.

30. Lacroix D, Sonnier M, Moncion A, Cheron G, Cresteil T. Expression of CYP3A in the human liver - evidence that the shift between CYP3A7 and CYP3A4 occurs immediately after birth. Eur J Biochem. 1997;247(2):625-634.

31. Treluyer JM, Gueret G, Cheron G, Sonnier M, Cresteil T. Developmental expression of CYP2C and CYP2C-dependent activities in the human liver: in-vivo/in-vitro correlation and inducibility. Pharmacogenetics. 1997;7(6):441-452.

32. Hill KD, Sampson MR, Li JS, Tunks RD, Schulman SR, CohenWolkowiez M. Pharmacokinetics of intravenous sildenafil in children with palliated single ventricle heart defects: effect of elevated hepatic pressures. Cardiol Young. 2015:1-9.

33. Muirhead GJ, Rance DJ, Walker DK, Wastall P. Comparative human pharmacokinetics and metabolism of single-dose oral and intravenous sildenafil. Br J Clin Pharmacol. 2002;53(Suppl 1):13S-20S

34. Muirhead GJ, Wilner K, Colburn W, Haug-Pihale G, Rouviex B. The effects of age and renal and hepatic impairment on the pharmacokinetics of sildenafil. Br J Clin Pharmacol. 2002;53(Suppl 1): 21S-30S.

35. Mukherjee A, Dombi T, Wittke B, Lalonde R. Population pharmacokinetics of sildenafil in term neonates: evidence of rapid maturation of metabolic clearance in the early postnatal period. Clin Pharmacol Ther. 2009;85(1):56-63.

36. Schwartz BG, Kloner RA. Drug interactions with phosphodiesterase-5 inhibitors used for the treatment of erectile dysfunction or pulmonary hypertension. Circulation. 2010;122(1):88-95. 
37. Burgess G, Hoogkamer H, Collings L, Dingemanse J. Mutual pharmacokinetic interactions between steady-state bosentan and sildenafil. Eur $J$ Clin Pharmacol. 2008;64(1):43-50.

38. Webb DJ, Freestone S, Allen MJ, Muirhead GJ. Sildenafil citrate and blood-pressure-lowering drugs: results of drug interaction studies with an organic nitrate and a calcium antagonist. Am J Cardiol. 1999; 83(5A):21C-28C.

39. Rubin LJ, Badesch DB, Fleming TR, et al. Long-term treatment with sildenafil citrate in pulmonary arterial hypertension: the SUPER-2 study. Chest. 2011;140(5):1274-1283.

40. Simonneau G, Rubin LJ, Galie N, et al. Addition of sildenafil to longterm intravenous epoprostenol therapy in patients with pulmonary arterial hypertension: a randomized trial. Ann Intern Med. 2008; 149(8):521-530.

41. Sastry BK, Narasimhan C, Reddy NK, Raju BS. Clinical efficacy of sildenafil in primary pulmonary hypertension: a randomized, placebo-controlled, double-blind, crossover study. J Am Coll Cardiol. 2004;43(7):1149-1153.

42. Galie N, Manes A, Negro L, Palazzini M, Bacchi-Reggiani ML, Branzi A. A meta-analysis of randomized controlled trials in pulmonary arterial hypertension. Eur Heart J. 2009;30(4):394-403.
43. Steinhorn RH, Kinsella JP, Pierce C, et al. Intravenous sildenafil in the treatment of neonates with persistent pulmonary hypertension. J Pediatr. 2009;155(6):841-847. e841.

44. Goldberg DJ, French B, McBride MG, et al. Impact of oral sildenafil on exercise performance in children and young adults after the Fontan operation: a randomized, double-blind, placebo-controlled, crossover trial. Circulation. 2011;123(11):1185-1193.

45. GiardiniA, BalducciA, Specchia S, Gargiulo G, Bonvicini M, Picchio FM. Effect of sildenafil on haemodynamic response to exercise and exercise capacity in Fontan patients. Eur Heart J. 2008;29(13):1681-1687.

46. Siehr SL, McCarthy EK, Ogawa MT, Feinstein JA. Reported sildenafil side effects in pediatric pulmonary hypertension patients. Front Pediatr. 2015;3:12.

47. US Food and Drug Administration. Revatio (sildenafil) label. Silver Spring, MD: US Food and Drug Administration; 2014. Available from: http://www.accessdata.fda.gov/drugsatfda_docs/label/2014/021845s01 1,022473s004,0203109s002lbl.pdf. Accessed December 10, 2015.
Drug, Healthcare and Patient Safety

\section{Publish your work in this journal}

Drug, Healthcare and Patient Safety is an international, peer-reviewed open-access journal exploring patient safety issues in the healthcare continuum from diagnostic and screening interventions through to treatment, drug therapy and surgery. The journal is characterized by the rapid reporting of reviews, original research, clinical, epidemiological and

\section{Dovepress}

post-marketing surveillance studies, risk management, health literacy and educational programs across all areas of healthcare delivery. The manuscript management system is completely online and includes a very quick and fair peer-review system. Visit http://www.dovepress.com/ testimonials.php to read real quotes from published authors.

Submit your manuscript here: http://www.dovepress.com/drug-healthcare-and-patient-safety-journal 